\title{
ARTICLES \\ THE ASYMPTOTIC EFFICIENCY OF LARGEST CLAIMS \\ REINSURANCE TREATIES
}

\author{
By ERHARD KREMER
}

Hamburg \& Löhnberg, FRG

\begin{abstract}
Reinsurance treaties defined as generalizations of the classical largest claims reinsurance covers are investigated with respect to the associated risk, defined as the variance of the insurer's retaining total claims amount. Instead of the unhandy variance corresponding handier asymptotic expressions are used. With these an asymptotic efficiency measure for comparing two such reinsurance covers is defined. It is shown that with respect to asymptotic efficiency the excess-of-loss treaty is better than the classical largest claims treaty. Furthermore the problem of giving optimal wheights to the ordered claims of a generalized largest claims cover is discussed.
\end{abstract}

\section{INTRODUCTION}

The choice of the appropriate treaty is a very old and fundamental problem in the reinsurance practice and theory. Already in the sixties actuaries discussed the problem of the optimal choice of a reinsurance treaty. The stop-loss and quota shares were shown to have some very interesting optimality properties (see e.g. Borch (1960), KAHN (1961), Lemaire (1973), OHLIN (1969), Pesonen (1967), Vajda (1962), Verbeek (1966) and the recent paper of Pesonen (1984)). Collective and individual treaties were compared and also an optimality property was given for the excess-of-loss treaty with respect to the class of individual treaties (see e.g. OHLIN (1969), GERBER (1980)). A short presentation of these results is given e.g. in KREMER (1986b).

Nearly nothing is known on the goodness of the largest claims reinsurance treaty or of some of its interesting generalizations, which are defined e.g. in KREMER (1986a), (1988a). Some remarks on certain dependencies between the largest claims and excess-of-loss treaty can be found in BERLINER (1972). Furthermore one knows that under certain conditions the net premium of the classical largest claims cover (see e.g. AMMETER (1964)) is asymptotically equivalent to the net premium of a corresponding excess-of-loss treaty plus additive term (see KREMER (1982)). A generalization of that result to the generalized largest claims reinsurance covers was given by the author (see KREMER (1984)) some years ago. Some more advanced results remain to show. In the following the author presents some first new investigations on the ASTIN BULLETIN, Vol. 20, No. 1 
goodness of the (classical or generalized) largest claims reinsurance treaties that are of the type one expects to get. Like in the already classical studies on the stop-loss, quota and excess-of-loss shares (see BORCH (1960), LeMAIRE (1973) and OHLIN (1969)) the author takes the inverse of the variance of the corresponding claims amount as measure for the goodness of the reinsurance treaty. Unfortunately one cannot give handy formulas for the variances under consideration. That's why the author replaces the variances by asymptotic formulas which were already cited in KREMER (1983). With these the asymptotic efficiency of two reinsurance treaties of the discussed type will be defined as the ratio of the inverses of the suitably transformed asymptotic variances. Like in the classical studies one takes the constraint that the net premiums of both treaties are (asymptotically) the same. With the help of this new concept of efficiency the classical largest claims cover (see AMMETER (1964)) is compared with the excess-of-loss treaty. Finally the author deals with the problem of choosing optimally coefficients, weighting the ordered claims in the generalized largest claims reinsurance treaty.

\section{THE GENERAL TREATY}

Consider a collective of insurance risks producing claims with sizes $X_{1}, X_{2}, X_{3}, \ldots$, each year. Denote with $N$ the random variable describing the number of claims per year. The claims sizes are assumed to be stochastically independent and identically distributed with distribution function $F$. Finally the claims number is assumed to be independent of the claims sizes. Investigated are reinsurance treaties which are based on the claims ordered in increasing amount i.e. on the random variables

$$
X_{N: 1} \leqq X_{N: 2} \leqq \ldots \leqq X_{N: N} .
$$

The reinsurance treaty conditions are defined by a family of weighting coefficients

$$
c_{n i}, \quad i=1,2, \ldots, n, \quad n=1,2,3 \ldots
$$

and a function

$h$ on the nonnegative reals .

With these quantities the part of the total claims amount

$$
S_{N}=\sum_{i=1}^{N} X_{i}
$$

that the insurer retains, when concluding the treaty, is given according

$$
R_{N}=\sum_{i=1}^{N} c_{N i} \cdot h\left(X_{N: i}\right) .
$$


Such a reinsurance cover was recently called linear reinsurance treaty based on ordered claims ( see e.g. KREMER (1988b)). In the more special situation where $h(x)=x$ holds for all $x$, one often denotes those reinsurance covers generalized largest claims reinsurance treaties (see e.g. KREMER 1988a)). The sense of the definition of the generalized treaty becomes obvious when considering some examples.

EXAmPLE 1. For the choice $c_{n i}=1$ for all $i=1,2,3, \ldots$ and $n=1,2,3, \ldots$ and the special function

$$
h(x)=\min (x, P)
$$

with a given nonnegative priority $P$ one gets the classical excess-of-loss treaty with priority $P$. The insurer has to pay for each claim up to the maximal amount $P . \nabla$

EXAMPLE 2. In case that $c_{n i}=1$ for all $i=1,2, \ldots, n-p$ and $c_{n i}=0$ otherwise and that $h(x)=x$ holds for all $x$, one has the (classical) largest claims reinsurance treaty, where the reinsurer pays for all claims except the $p$ largest ones. $\nabla$

The reader is invited to give some more examples, e.g. one can combine the situations of the example 1 and 2 . Notice that in the present investigations we consider the claims amount remaining by the insurer and not like in the previous studies (see KREMER (1986a), (1988a), (1988b)) the claims amount taken by the reinsurer. In other words, the $R_{N}$ here is just the $S_{N}-R_{N}$ of the previous papers.

\section{THE ASYMPTOTIC EFFICIENCY}

Obviously the class of linear reinsurance treaties based on ordered claims is fairly large. One can choose among many different such reinsurance covers. The question appears which of two given different treaties is preferable. For deciding, one needs an appropriate measure with which one can select the treaty which is more advantageous. A classical measure for judging the goodness of a reinsurance treaty is the variance of the total claims amount under consideration while choosing some parameters of the treaty such that the mean value of the total claims amount is fixed (see e.g. OHLIN (1969) and KREMER (1986b) chapter 5.1). In case of our above defined linear reinsurance treaty based on ordered claims no handy expressions for the expectation $E\left(R_{N}\right)$ and variance $\operatorname{Var}\left(R_{N}\right)$ exist in general. Fortunately one can give elegant formulas for both quantities with asymptotic considerations. More concretely the author gave in a previous paper (see KREMER (1983)) expressions for 
$E\left(R_{N}\right)$ and $\operatorname{Var}\left(R_{N}\right)$ that are asymptotically equivalent to both quantities. These results are basic to all that follows and will be presented in the sequel.

Consider a sequence of growing collectives, indexed with the integer $k=1,2,3, \ldots$ Denote with $N_{k}$ the claims number of the collective no. $k$ and suppose that

$$
\begin{aligned}
& \lim _{k \rightarrow \infty}\left(E\left(N_{k}\right)\right)=+\infty \\
& \lim _{k \rightarrow \infty}\left(\frac{\operatorname{Var}\left(N_{k}\right)}{E\left(N_{k}\right)}\right)=c
\end{aligned}
$$

with an arbitrary, but fixed constant $c$. The random variables of the claims amounts are the same in each collective and denoted by the variables

$$
X_{1}, X_{2}, X_{3}, \ldots
$$

They are assumed to satisfy the conditions given in the beginning of the previous section, especially they are assumed to be stochastically independent of the claims numbers $N_{k}$ and to have the distribution function $F$. The linear reinsurance treaty based on ordered claims now depends also on the collective number $k$, more concretely the weighting coefficients are dependent of the index number $k$ :

$$
c_{n i}=c_{n i}^{(k)}, \quad \text { with } \quad k=1,2,3, \ldots,
$$

whereas the function $h$ is independent of the number $k$. For giving the asymptotic formulas for the expectation and variance of the claims amount

$$
R_{N}=R_{N_{k}}^{(k)}=\sum_{i=1}^{N_{k}} c_{N_{k} i}^{(k)} \cdot h\left(X_{N_{k}: i}\right)
$$

one defines the family of functions

$$
b_{n}^{(k)}, \quad n=1,2,3, \ldots, \quad k=1,2,3, \ldots
$$

according

$$
\begin{aligned}
b_{n}^{(k)}(0) & =c_{n 1}^{(k)}, \\
b_{n}^{(k)}(u) & =c_{n i}^{(k)} \text { for } u \text { in the interval } \quad((i-1) / n, i / n] \quad \text { and } \quad i=1,2,3, \ldots, n,
\end{aligned}
$$

and assumes that there exist an asymptotic weighting function $b$ and numbers

$$
t_{i}, \quad i=0,1, \ldots, m+1
$$


with $t_{i}<t_{i+1}, t_{0}=0, t_{m+1}=1$ such that

$$
\lim _{k \rightarrow \infty}\left(b_{n_{k}}^{(k)}\right)=b
$$

uniformly on closed subintervals of the complementary set of $\left\{t_{1}, \ldots, t_{m}\right\}$ and for each sequence $\left(n_{k}, k=1,2, \ldots\right)$ satisfying

$$
\lim _{k \rightarrow \infty}\left(\frac{n_{k}}{E\left(N_{k}\right)}\right)=1 \text {. }
$$

The function $b$ is supposed to consist of two parts

$$
b=b_{s}+b_{d}
$$

where $b_{s}$ is of bounded variation and continuously differentiable and $b_{d}$ is a step function with steps at the points $t_{1}, \ldots, t_{m}$. Finally the function $h$ shall be nondecreasing, $F$ be continuous and strictly increasing (from both sides) at the points $F^{-1}\left(t_{i}\right), i=1,2, \ldots, m$, with the convention

$$
F^{-1}(u)=\inf \{x: F(x) \geqq u\} .
$$

With all these notations and assumptions one has the important result that with the expressions

$$
\begin{aligned}
\mu_{F}(b, h)= & \int_{0}^{\infty} b(F(x)) \cdot h(x) F(d x) \\
\sigma_{F}^{2}(b, h)= & \int_{0}^{\infty} \int_{0}^{\infty}(\min (F(s), F(t))-F(s) \cdot F(t)) \times \\
& \times b(F(s)) \cdot b(F(t)) h(\mathrm{ds}) h(d t)
\end{aligned}
$$

holds

$$
\begin{aligned}
& \lim _{k \rightarrow \infty}\left(\frac{E\left(R_{N_{k}}^{(k)}\right)}{E\left(N_{k}\right)}\right)=\mu_{F}(b, h) \\
& \lim _{k \rightarrow \infty}\left(\frac{\operatorname{Var}\left(R_{N_{k}}^{(k)}\right)}{E\left(N_{k}\right)}\right)=\sigma_{F}^{2}(b, h)+c \cdot \mu_{F}^{2}(b, h)
\end{aligned}
$$

(see Theorem 1 in KREMER (1983)). In these formulas the distribution function $F$ is fixed and given. The expressions depend only through the functions $b$ and $h$ on the linear reinsurance treaty based on ordered claims.

Now coming back to judging the goodness of a linear reinsurance treaty based on ordered claims. In the classical approach of comparing reinsurance 
treaties one fixes the expectation of the claims amount under consideration and investigates the corresponding variance. According to the elegant result (3) the fixing of the expectation can be formulated in an asymptotic sense according:

$$
\mu_{F}(b, h)=\text { constant } .
$$

Then according to (4) the investigation of the variance can be expressed in an asymptotic sense as the investigation of the expression

$$
\sigma_{F}^{2}(b, h) .
$$

All above remarks now are summarized in the following definition.

DEFINITION. In the above setting consider two linear reinsurance treaties based on ordered claims with corresponding functions $b_{i}, h_{i}, i=1,2$. Suppose that

$$
\mu_{F}\left(b_{1}, h_{1}\right)=\mu_{F}\left(b_{2}, h_{2}\right)
$$

is satisfied. Then the value

$$
\operatorname{EFF}_{F}(1: 2)=\left(\frac{\sigma_{F}^{2}\left(b_{2}, h_{2}\right)}{\sigma_{F}^{2}\left(b_{1}, h_{1}\right)}\right)
$$

is called asymptotic efficiency of the treaty no. 1 relative to the treaty no. 2. In case that

$$
\operatorname{EFF}_{F}(1: 2)>1
$$

holds true, the treaty no. 1 is called better than the treaty no. 2 (at the underlying claims size distribution function $F$ ). In case that in (6) one has equality, both treaties are called to be (asymptotically) equivalent. $\nabla$

Obviously this definition gives a practicable formal instrument for comparing the linear reinsurance treaties based on ordered claims. In case of the socalled generalized largest claims treaties (see above) one has $h(x)=x$ for all $x$, so that $\mu_{F}, \sigma_{F}^{2}$ depend only on $b$. Then write shorter $\mu_{F}(b), \sigma_{F}^{2}(b)$ for the special $\mu_{F}(b, h), \sigma_{F}^{2}(b, h)$. For illustration an important example shall be discussed.

\section{AN EXAMPLE}

Consider the (classical) largest claims reinsurance treaty of the example 2 in the above context of growing collectives. Denote the number $p$ of the treaty in the collective no. $k$ by $p_{k}$. Assume that

$$
\lim _{k \rightarrow \infty}\left(\frac{p_{k}}{E\left(N_{k}\right)}\right)=s,
$$


for an arbitrary, fixed value $s$ between zero and one. This treaty shall be compared with the excess-of-loss treaty with priority $P>0$ (see the example 1). With these notations one gets the corresponding functions:

(a) for the excess-of-loss treaty:

$$
\begin{gathered}
b_{1}(u)=1, \quad \text { for all } u, \\
h_{1}(x)=\min (x, P), \text { for all } x,
\end{gathered}
$$

(b) for the largest claims treaty:

$$
\begin{aligned}
b_{2}(u) & =1, \text { if } u \text { is smaller or equal to } 1-s, \\
& =0, \text { elsewhere, } \\
h_{2}(x) & =x, \text { for all } x,
\end{aligned}
$$

and the interesting result:

THEOREM. The excess-of loss treaty is better than the largest claims cover in the just given setting. $\nabla$

Proof. Since

$$
\begin{aligned}
& \mu_{F}\left(b_{1}, h_{1}\right)=\int_{[0, P]} x F(d x)+P \cdot(1-F(P)) \\
& \mu_{F}\left(b_{2}, h_{2}\right)=\int_{\left[0, F^{-1}(1-s)\right]} x F(d x),
\end{aligned}
$$

the equation (5) means nothing else but that:

$$
\int_{\left[0, P^{\prime}\right]} x F(d x)-\int_{[0, P]} x F(d x)=P \cdot(1-F(P))
$$

with the priority $P^{\prime}=F^{-1}(1-s)$. This implies at once that $P^{\prime} \geqq P$. Since $F\left(P^{\prime}\right)<1$, one also has $F(P)<1$. Consequently one has because of $(7)$ the stronger condition:

$$
P^{\prime}>P \text {. }
$$

Inserting the $b_{i}, h_{i}$ into the expression (2) implies the in structure identical formulas: 


$$
\begin{aligned}
& \sigma_{F}^{2}\left(b_{1}, h_{1}\right)=2 \cdot \int_{0}^{P} F(r) \cdot \int_{r}^{P}(1-F(t)) d t d r \\
& \sigma_{F}^{2}\left(b_{2}, h_{2}\right)=2 \cdot \int_{0}^{P^{\prime}} F(r) \cdot \int_{r}^{P^{\prime}}(1-F(t)) d t d r,
\end{aligned}
$$

from which one concludes easily with (8) that the excess-of-loss treaty is the better one. $\nabla$

This result can be seen as a theoretical justification for the common preference of the excess-of-loss treaty on the international reinsurance market. Surprisingly the proof of the theorem is fairly simple, when using the concepts of the preceeding section. The result is new and fits well to the investigations of BerLINER (1972). For given $s \in(0,1)$ and distribution function $F$ one can easily compute the efficiency of the largest claims treaty relative to the excess-of-loss treaty. Since $s$ is given, the $P^{\prime}$ is fixed, so that one can compute the corresponding priority $P$ from the equation (7). For computing $E F F_{F}(1: 2)$ it then remains to evaluate the integrals in (9) and (10) and then to take the ratio. Exemplarily one can take for $F$ the classical Pareto-model.

Corollary. Suppose that $F$ is the Pareto-model, i.e.

$$
F(x)=1-x^{-a} \text {, for } x \text { larger than } 1,
$$

with a given parameter a larger than 2 . Define the following function:

$$
\begin{gathered}
g(y)=y^{2 \cdot(1-a)} \cdot\left(\frac{1}{2 \cdot(a-1)}\right)+y^{2-a} \cdot\left(\frac{a-1}{a-2}\right)- \\
-y^{1-a} \cdot\left(\frac{a}{a-1}\right)-\frac{a}{2 \cdot(a-1) \cdot(a-2)}
\end{gathered}
$$

and the values:

$$
\begin{aligned}
& y 1=a^{1 /(1-a)} \cdot s^{-1 / a} \\
& y 2=s^{-1 / a} .
\end{aligned}
$$

With this notation one gets for the asymptotic efficiency of the largest claims relative to the excess-of-loss treaty the result:

$$
\operatorname{EFF}_{F}(1: 2)=\frac{g(y 1)}{g(y 2)},
$$

in case that $s$ is smaller than $a^{a /(1-a)}$. 
Proof. One evaluates with routine calculations the equations (7), (9) and (10). More concretely (7) means with $y 1=P, y 2=P^{\prime}$ that:

$$
a \cdot \int_{y 1}^{y 2} x^{-a} d x=y 1^{1-a}
$$

what is equivalent with

$$
y 1=a^{1 /(1-a)} \cdot y 2 .
$$

Since :

$$
P^{\prime}=F^{-1}(1-s)=s^{-1 / a},
$$

one has the formulas for $y 1=P$ and $y 2=P^{\prime}$. Furthermore one shows that:

$$
\begin{aligned}
& \int_{0}^{y} F(r) \cdot \int_{r}^{y}(1-F(t)) d t d r \\
& \quad=\int_{1}^{y}\left(1-r^{-a}\right) \cdot\left(\int_{r}^{y} t^{-a} d t\right) d r=\ldots=g(y) \cdot(1-a)^{-1} \cdot \nabla
\end{aligned}
$$

The Corollary shows that the efficiency depends for given parameters of the distribution function $F$ solely on the value of $s$.

\section{OPTIMAL WEIGHTS}

In this section the problem of how to choose the weighting coefficients $c_{n i}$, $i=1,2, \ldots, n, n=1,2, \ldots$ is discussed for the situation that the function $h$ of the linear reinsurance treaty based on ordered claims is given and the insurer likes to retain a net premium exceeding a minimum amount $\mu$. Without loss of generality let us assume that

$$
h(x)=x, \quad \text { for all } x .
$$

This means that one deals with the generalized largest claims reinsurance treaty and tries to find some in some sense optimal weighting coefficients for given claims size distribution function $F$ and on a constraint on the insurer's net income. Suppose that one has one in some sense optimal asymptotic weighting function $b$ for the treaty. Then an adequate choice of the weighting coefficients is to take

$$
\begin{aligned}
c_{n i}=b\left(\frac{i}{n}\right), \quad \text { for } \quad i & =1,2, \ldots, n \\
n & =1,2, \ldots .
\end{aligned}
$$


With this choice the treaty is in some sense asymptotically optimal. So the problem of giving adequate weighting coefficients reduces to the problem of determining an optimal asymptotic weighting function. The above presented concepts and ideas make it possible to define what might be regarded as an optimal asymptotic weighting function $b$.

DeFinition. Consider the class of generalized largest claims reinsurance treaties with asymptotic weighting functions $b$ in the above context of growing collectives. Suppose that one has with a given constant $\mu$ the restriction on $b$ :

$$
\mu_{F}(b) \geqq \mu,
$$

where $F$ is the fixed underlying distribution function of the claims sizes of the collective. The asymptotic weighting function $b_{*}$ is called optimal in the class $\Delta$ if:

$$
\bar{\sigma}_{F}^{2}\left(b_{*}\right)=\inf _{b \in \Delta}\left[\bar{\sigma}_{F}^{2}(b)\right],
$$

where $\Delta$ is a given class of asymptotic weighting functions with each $b \in \Delta$ satisfying (11) and the $\bar{\sigma}_{F}^{2}(b)$ is the right hand side of (4). A treaty with corresponding asymptotic weighting function $b_{*}$ is called asymptotically optimal in the class of treaties with weighting functions $b \in \Delta . \nabla$

Assuming that the basic claims size distribution function $F$ is continuous, one can reformulate $\mu_{F}(b)$ and $\sigma_{F}^{2}(b)$ according:

$$
\begin{gathered}
\mu_{F}(b)=\int_{0}^{1} b(u) \cdot F^{-1}(u) d u \\
\sigma_{F}^{2}(b)=\iint(\min (u, v)-u \cdot v) \cdot b(u) \cdot b(v) F^{-1}(d u) F^{-1}(d v) .
\end{gathered}
$$

Obviously $\sigma_{F}^{2}$ is something like a quadratic form and the $\mu_{F}$ nothing else but a linear functional on the set of all asymptotic weighting functions $b$. In case one restricts to uniformly continuous functions $b$, the determination of an optimal $b$, reduces to a typical infinite optimization problem, i.e. to the minimization of the sum of a quadratic and a squared linear form under the contraint that a linear functional exceeds a given constant. For solving one can apply results of the socalled infinite optimization theory. The reader is referred to the literature on this mathematical topic ( see e.g. KRABS (1975)). Because of practical reasons one will take in addition the condition on $b$ that

$$
\begin{aligned}
& b(u) \quad \text { is nonnegative for all } u \text { and } \\
& \text { bounded by the amount } 1 .
\end{aligned}
$$


One knows that the optimal $b_{*}$ can be determined such that with a nonnegative $\lambda_{*}$ the tupel $\left(b_{*}, \lambda_{*}\right)$ is a saddlepoint of the Lagrange-function

$$
L(b, \lambda)=\bar{\sigma}_{F}^{2}(b)-\lambda \cdot\left(\mu_{F}(b)-\mu\right)
$$

with respect to all nonnegative $b$ and $\lambda$.

In practice one does not know $F$ but only knows the corresponding empirical distribution function $F_{m}$, defined with the known $m$ past claims amounts $X_{1}, X_{2}, \ldots, X_{m}$ according:

$$
F_{m}(x)=\left(\frac{1}{m}\right) \cdot\left(\text { number of } X_{i} \leqq x\right) .
$$

Then one clearly inserts $F_{m}$ for $F$ in the $\mu_{F}(b), \sigma_{F}^{2}(b)$, yielding as results:

$$
\begin{aligned}
\mu_{F_{m}}(b)= & \left(\frac{1}{m}\right) \cdot \sum_{i=1}^{m} b\left(\frac{i}{m}\right) \cdot X_{m: i} \\
\sigma_{F_{m}}^{2}(b)= & \left.\left(\frac{1}{m^{2}}\right) \cdot \sum_{i=1}^{m-1} \sum_{j=1}^{m-1}(m \cdot \min (i, j)-i \cdot j)\right) \times \\
& \times b\left(\frac{i}{m}\right) \cdot b\left(\frac{j}{m}\right) \cdot\left(X_{m:(i+1)}-X_{m: i}\right) \cdot\left(X_{m:(j+1)}-X_{m: j}\right)
\end{aligned}
$$

with probability one. Here one uses that for continuous $F$ the ordered claims are all different, i.e. :

$$
X_{m: 1}<X_{m: 2}<\ldots<X_{m: m},
$$

with probability one. In case some ordered claims are equal, both expressions in (12), (13) have to be modified slightly. Let us restrict exemplarily to the situation (14). In (12), (13) the asymptotic weighting function $b(u)$ appears only at the points $u=i / m$, where $i$ runs from 1 to $m$. One can calculate optimal values $b_{1}, b_{2}, \ldots, b_{m}$ for $b(1 / m), b(2 / m), \ldots, b(1)$ by minimizing $\bar{\sigma}_{F_{m}}^{2}(b)$ with respect to the $b(i / m)$ (with $i=1,2, \ldots, m$ ) under the constraints that

$$
\mu_{F_{m}}(b) \geqq \mu \text {. }
$$

and that the $b(i / m)$ are nonnegative and bounded by 1 . This is nothing but a standard problem of the (finite) optimization theory, which can be solved with the methods of the quadratic programming (see e.g. KÜNZI et al. (1967)).

Having calculated the optimal values $b_{*}(i / m)=b_{i}$ with $i=1,2, \ldots, m$, one likes to have also values $b_{*}(u)$ for the $u$ unequal to the $i / m$ with $i=1,2, \ldots, m$. A practical approach might the simply to interpolate and extrapolate the function $b_{*}(u)$ between and from the points $u=i / m$ with $i=1,2, \ldots, m$, by using a suitable method of the numerical mathematics. 
Since the methods of the quadratic programming nowadays work without great problems on each modern computer, one can determine with the given procedure an approximate optimal asymptotic weighting function $b_{*}$ in case one has the empirical distribution function.

Clearly the problem of giving optimal weights $c_{n i}(i=1,2, \ldots, n$, $n=1,2, \ldots$ ) or more concretely an optimal asymptotic weighting function $b$ is mainly of theoretical interest. In practice the reinsurer clearly will not loose time with computing such an "optimal" reinsurance treaty. So the author restricts to the above short discussion and closes the paper.

\section{REFERENCES}

Ammeter, H. (1964) The rating of "largest claim" reinsurance covers. Quaterly letter from the allgemeene reinsurance companies , 79-109.

Beard, R., Pentikänen, P. and Pesonen, E. (1969) Risk Theory. Chapman \& Hall, Londen.

BERliner, B. (1972) Correlations between excess-of-loss reinsurance covers and reinsurance of the $n$ largest claims. ASTIN Bulletin, 260-275.

Borch, K. (1960), An attempt to determine the optimum amount of stop loss reinsurance. Transactions of the XVI international congress of actuaries, 597-610.

GERBER, H.U. (1980) An introduction to mathematical risk theory. S.S. Huebner foundation, Philadelphia.

KAHN, P. (1961) Some remarks on a recent paper by Borch. ASTIN Bulletin, 265-272.

KraBS, W. (1975) Optimierung und Approximation. Teubner Studientexte, Stuttgart.

KREMER, E. (1982), Rating of largest claims and ECOMOR reinsurance treaties for large portfolios. ASTIN Bulletin, 47-56.

KREMER, E. (1983), Rating of nonproportional reinsurance treaties based on ordered claims. A lecture given at the NATO-study institute in Leuven. Published in 1984 in the NATO-ASI series: Premium Calculation in Insurance, edited by Goovaerts, M., De Vylder, F. and Haezendonck, J.

KREMER, E. (1984) An asymptotic formula for the net premium of some reinsurance treaties. Scandinavian Actuarial Journal, 11-22.

KREMER, E. (1986a) Recursive calculation of the net premium of largest claims reinsurance covers. ASTIN Bulletin, 101-108.

KREMER, E. (1986b) Höhere Versicherungsmathematik. Verein zur Förderung der angewandten mathematischen Statistik und Risikotheorie. Hamburg.

Kremer, E. (1988a) A general bound for the net premium of largest claims reinsurance treaties. ASTIN Bulletin, $69-78$.

KREMER, E. (1988b) Net premiums of nonproportional reinsurance covers in nonlife insurance. Verein zur Förderung der angewandten mathematischen Statistik und Risikotheorie. Hamburg.

KUnZI, H.P., Tzschach, H.G. and Zehnder, C. A. (1967) Mathematische Optimierung. Teubner Studienbücher, Stuttgart.

Lemaire, J. (1973) Sur la détermination d'un contract optimal de réassurance. ASTIN Bulletin, 165180 .

OHLIN, J. (1969) On a class of measures of dispersion with application to optimal reinsurance. ASTIN Bulletin, 249--266.

Pesonen, E. (1967) On optimal properties of the stop loss reinsurance. ASTIN Bulletin, 175-176. Pesonen, M. I. (1984) Optimal reinsurances. Scandinavian Actuarial Journal, 65-90.

VAJDA, S. (1962) Minimum variance reinsurance. ASTIN Bulletin, 257-260.

VERBEEK, H. G. (1966) On optimal reinsurance. ASTIN Bulletin, 29-38.

\section{Prof. Dr. ERHARD KREMER}

Verein zur Förderung der Angewandten Mathematischen Statistik und Risikotheorie

Research \& Relax Area, Wallstr. 15, 6293 Löhnberg 1, FRG. 